\title{
Re-operation within 30 days of radical cystectomy: Identifying high-risk patients and complications using ACS-NSQIP database
}

Rashid K. Sayyid, MD, MSc${ }^{1}$; Diana Magee, MD, $\mathrm{MPH}^{2}$; Amanda E. Hird, MD²; Benjamin T. Harper, MD ${ }^{1}$; Eric Webb, MD ${ }^{1}$; Katherine L. Fratino, MD ${ }^{1}$; Martha K. Terris, MD ${ }^{1,3}$; Rabii Madi, MD, MBA ${ }^{1,3}$; Raj Satkunasivam, MD, MS ${ }^{4}$; Christopher J.D. Wallis, $\mathrm{MD}, \mathrm{PhD}^{2}$; Zachary Klaassen, $\mathrm{MD}, \mathrm{MSc}^{1,3}$

${ }^{1}$ Section of Urology, Department of Surgery, Medical College of Georgia-Augusta University, Augusta, GA, United States; ${ }^{2}$ Division of Urology, Department of Surgery, University of Toronto, Toronto, ON, Canada; ${ }^{3}$ Georgia Cancer Center, Augusta, GA, United States; ${ }^{4}$ Department of Urology and Center for Outcomes Research, Houston Methodist Hospital, Houston, TX, United States

Cite as: Can Urol Assoc J 2020 July 17; Epub ahead of print. http://dx.doi.org/10.5489/cuaj.6490

Published online July 17, 2020

$* * *$

\section{Abstract}

Introduction: Radical cystectomy (RC) is a highly morbid procedure, with 30-day complication rates approaching $31 \%$. Our objective was to determine risk factors for re-operation within 30 days following a $\mathrm{RC}$ for non-metastatic bladder cancer.

Methods: We included all patients who underwent a RC for non-metastatic bladder cancer using The American College of Surgeons National Surgical Quality Improvement Program database between January 1, 2007 and December 31, 2014. Logistic regression analyses were used to evaluate predictors of re-operation.

Results: A total of 2608 patients were included; $5.8 \%$ of patients underwent reoperation within 30 days. On multivariable analysis, increasing body mass index (BMI) (odds ratio [OR] 1.04; 95\% confidence interval [CI] 1.01-1.07), AfricanAmerican race (vs. Caucasian OR 2.29; 95\% CI 1.21-4.34), and history of chronic obstructive pulmonary disease (COPD) (OR 2.33; 95\% CI 1.45-3.74) were significant predictors of re-operation within 30 days of RC. Urinary diversion type (ileal conduit vs. continent) and history of chemotherapy or radiotherapy within 30 days prior to RC were not. Patients who underwent re-operation within this timeframe had a significantly higher mortality rate $(4.0 \%$ vs. $1.6 \%)$ and were more likely to experience cardiac ( $7.2 \%$ vs. $1.9 \%)$, pulmonary $(23.0 \%$ vs. $3.0 \%)$, neurological $(2.0 \%$ vs. $0.49 \%$ ), and venous thromboembolic events (10.5\% vs. $5.4 \%)$, as well as infectious complications $(64.5 \%$ vs. $24.1 \%)$ with a significantly longer hospital length of stay (16.5 vs. 7.0 days).

Conclusions: Recognizing increasing BMI, COPD, and African-American race as risk factors for re-operation within 30 days of $\mathrm{RC}$ will allow urologists to 
preoperatively identify such high-risk patients and prompt them to adopt more aggressive approaches to minimize postoperative surgical complications.

\section{Introduction}

Radical cystectomy (RC) with pelvic lymph node dissection remains the gold standard treatment for non-metastatic, muscle-invasive and high-risk, non-muscle invasive bladder tumors, such as BCG-refractory tumors. ${ }^{1,2}$ This procedure however is highly morbid, with 30-day overall complication, transfusion, prolonged hospitalization, readmission, and perioperative mortality rates reported at $31.1 \%$, $24.4 \%, 25.9 \%, 20.2 \%$, and $2.7 \%$, respectively. ${ }^{3}$ Reoperation within 30 days of RC is a notable complication that may be associated with higher wound complications rates, hospital length stay, and mortality rates. ${ }^{4}$ This underlies the significant health and financial consequences of this complication. Efforts to identify patients at higher risk of reoperation within 30 days of $\mathrm{RC}$, particularly at a multi-institutional level, are thus needed in order to implement pre- and post-op strategies to minimize this complication. Our goal was to identify predictors of reoperation within 30 days of RC using a national, validated database.

\section{Methods}

\section{Study design, setting, and participants}

We utilized The American College of Surgeons National Surgical Quality Improvement Program Database (NSQIP), which is a nationally validated, riskadjusted, outcomes-based program, ${ }^{5}$ to identify patients undergoing an RC (Common Procedural Terminology code: $51590,51595,51596)$ with a post-procedural diagnosis of bladder cancer (International Classification of Diseases-9 code: 188.x), between January 1, 2007 and December 31, 2014. Patients missing important baseline characteristics and outcome measures were excluded from the cohort. This study was exempt from required ethics board approval at our institutions.

\section{Study outcomes}

The primary study outcome was reoperation within 30 days of RC. Of note, reason for or type of reoperation performed was not available for this dataset. Secondary outcomes were adverse events within 30 days of surgery, including: mortality, cardiac, neurologic, pulmonary, venous thromboembolic, and infectious events, and prolonged hospital length of stay (i.e. greater than 7 days).

\section{Study variables}

Baseline patient characteristics were abstracted from the NSQIP database and included: patient age, gender, race, body mass index (BMI), American Society of Anesthesiologists (ASA) physical status classification system score, history of 
cardiac, neurologic, chronic obstructive pulmonary disease (COPD), and diabetes mellitus diagnoses, need for dialysis, active smoking, chronic steroid use, pre-op chemotherapy or radiotherapy (both within 90 days of surgery), pre-op serum albumin (surrogate for pre-op nutritional status), pre-op functional status (independent vs. partially/totally dependent), and type of diversion created (ileal conduit vs. continent diversion). Pathologic information was not available for this dataset.

\section{Statistical methods}

Continuous variables were reported using medians and interquartile ranges, while categorical variables were described using proportions. Univariable analysis was performed using the Chi-Square and Fisher's exact test, where appropriate, for categorical variables, with the Student's t-test and Wilcoxon-Rank Sum test used to compare continuous variables. Multivariate logistic regression analysis accounting for clinical factors identified a priori as potentially influencing rates of reoperation (age, gender, BMI, race, ASA score, history of DM, history of COPD, active smoking, chronic steroid use, functional status, and type of diversion) was used to evaluate predictors/risk factors for reoperation within 30 days of RC. All statistical analysis was performed using SAS 9.4 (SAS Institute Inc., Cary, NC, USA).

\section{Results}

We identified 2,608 patients undergoing RC for bladder cancer during the study time frame. Median patient age was 69.0 years (IQR $61.0-76.0$ ). 82.4\% of patients were males, $89.0 \%$ were Caucasian, and $79.5 \%$ received an ileal conduit at time of surgery. 152 patients $(5.8 \%)$ underwent reoperation within 30 days of $\mathrm{RC}$.

On univariate analysis, race (10.9\% of African-Americans vs. $5.1 \%$ of Caucasians and $4.0 \%$ of other races, $\mathrm{p}=0.04)$, history of COPD $(12.3 \%$ vs. $5.3 \%$, $\mathrm{p}<0.01)$, and higher BMI $\left(29.1 \mathrm{~kg} / \mathrm{m}^{2}\right.$ for those with reoperation within 30 days vs. $27.8, \mathrm{p}=0.017$ ) were associated with higher reoperation rates. Choice of urinary diversion, pre-operative chemotherapy or radiotherapy use, serum albumin, gender, ASA score, cardiac, neurologic, and diabetes mellitus histories, need for dialysis, active smoking, chronic steroid use, and pre-operative functional status were not significantly different between patients who did and did not undergo reoperation within 30 days of RC (Table 1).

On multivariable analysis, increasing BMI (OR 1.04, 95\%CI 1.01-1.07), African American race (vs Caucasian OR 2.29, 95\%CI 1.21-4.34), and history of COPD (OR 2.33, 95\%CI 1.45-3.74) were the only significant predictors for higher risk of reoperation within 30 days of $\mathrm{RC}$ (Table 2).

Patients who underwent reoperation were significantly more likely to experience the following adverse events within 30 days of RC: mortality ( $4.0 \% \mathrm{vs}$. $1.6 \%)$, cardiac $(7.2 \%$ vs. $1.9 \%)$, neurologic $(2.0 \%$ vs. $0.5 \%)$, pulmonary $(23.0 \%$ vs. $3.0 \%)$, and venous thromboembolic events (10.5\% vs. $5.4 \%)$, sepsis $(43.4 \%$ vs. $11.9 \%$ ), pneumonia (13.2\% vs. $2.8 \%$ ), wound infections ( $48.0 \%$ vs. $11.0 \%)$, and 
prolonged hospital length of stay, with median length of stay of 16.5 days (IQR 8.026.5) compared to 7.0 days (6.0-10.0) for patients who did not undergo reoperation.

\section{Discussion}

Based on this large, multi-institutional cohort, cystectomy, more than 1 in 20 patients (5.8\%) undergoing radical cystectomy for bladder cancer will undergo reoperation within 30 days. Patients who are African-American, have COPD, and have a higher BMI experience an increased likelihood of reoperation. Such reoperation is associated with a 2.5 fold increased risk of death, a 3.8 fold increased risk of experiencing a cardiac event, and a significantly longer hospital length of stay (16.5 vs. 7.0 days), which highlights the health and financial consequences of this worrisome complication.

Multiple studies have evaluated the impact of obesity on post-operative complications following a radical cystectomy, with Arora et al. and Al-Daghmin et al. demonstrating that increasing obesity was associated with a progressively worsening 30-day complication rate ${ }^{6}$ and a higher re-admission rate, ${ }^{7}$ respectively. Interestingly, $13.4 \%$ of obese patients had hypoalbuminemia, ${ }^{6}$ a marker of malnutrition.

Hypoalbuminemia was shown to be independently associated with a 2.33 fold higher mortality rate, ${ }^{6}$ yet in our study there were no significant differences in albumin levels between the two groups. Future studies that investigate the significance of other pre-operative nutritional markers such as pre-albumin, a non-specific marker of shortterm nutritional status, ${ }^{8}$ may further highlight the significance of pre-operative nutritional status on post-RC outcomes.

A history of COPD was one of the most prominent predictors of reoperation following RC, with a 2.33 -fold increased risk of reoperation. Such patients are classically underweight, malnourished, have poor pulmonary reserve, ${ }^{9}$ and poor exercise tolerance, ${ }^{10}$ which predispose to poor post-operative outcomes. Moreover these patients may have a chronic cough, which may mechanically contribute to poor wound healing. Pre-operative rehabilitation/exercise programs for patients with COPD have been proposed by anesthesiologists in an effort to "optimize" respiratory status. ${ }^{10}$ This highlights the importance of multispecialty collaboration in the preoperative setting to improve post-operative outcomes. Notably, active smokers did not have significantly increased reoperation rates in our cohort, although this is likely a result of our study sample size/power, as the OR was 1.46 with a $95 \%$ CI of 0.99 2.17. Smoking has been previously shown to be associated with increased incidence of Clavien II-V complications following RC. ${ }^{11}$

Interestingly, African-American race was found to be a significant risk factor for reoperation following $\mathrm{RC}$, even after adjusting for medical comorbidities with multivariate analysis. African-Americans have been shown to have higher clinical grade and stage bladder tumors at presentation, with increased risk of lymph node involvement and metastatic disease. ${ }^{12,13}$ Furthermore, there is evidence of racial disparities in the quality of medical care, particularly for bladder cancer patients,. African-Americans, for example, are less likely to receive neoadjuvant chemotherapy 
within eight weeks of muscle-invasive bladder cancer diagnosis, which has been shown to be associated with higher risk of tumor upstaging. ${ }^{14}$ This combination of biological and resource utilization differences may explain the increased risk of adverse outcomes in African-American patients, independent of their medical comorbidities.

Pre-operative receipt of chemotherapy or radiotherapy within 90 days of RC was notably not a significant predictor of re-operation. This is consistent with results published by Johnson et al. whereby they demonstrated that neoadjuvant chemotherapy prior to RC does not increase the risk of perioperative morbidity, including re-operation rate. ${ }^{15}$ There may be an element of selection bias in these studies as patients who may seem "fitter" per the physician's judgment are more likely to be referred for pre-operative chemotherapy, and these are the patients that are likely to fare better post-operatively.

Our results are consistent with a previously published report investigating perioperative reoperation rates following RC. The reported 90-day reoperation rate was $5.7 \%$, with fascial dehiscence (29\%), bowel obstruction (21\%), and enteric anastomotic leak $(8 \%)$ the most common reasons for reoperation. ${ }^{4}$ Increasing BMI was also found to be a predictor of reoperation, ${ }^{4}$ however this study did not highlight the significance of race or COPD as predictors of reoperation. Furthermore, the population was from a single institution, which limits the generalizability of these results when compared to data from a national, validated database such as ACSNSQIP.

The rate of adverse events for patients who underwent reoperation within 30 days of RC was alarmingly high. In addition to the obvious health burden of such complications, the increased financial burden needs be emphasized. It has been consistently shown that patients with major post-operative complications following abdominal surgery have almost two fold increased direct hospital costs $(\$ 56,224$ vs $\$ 29,038) .{ }^{16}$ Thus, the need for pre- and immediate post-operative efforts, such as preoperative pulmonary rehabilitation programs,${ }^{10}$ extensive counseling regarding importance of weight loss and smoking cessation, and Enhanced Recovery After Surgery (ERAS) pathways, ${ }^{17}$ to minimize post-operative complications in high-risk patients, and thus limit financial costs is crucial in this setting.

This study is limited by its retrospective nature. We were also unable to evaluate the reason for or type of reoperation. Pre-op pathologic information was not available, and this may have influenced the surgeon's surgical approach, leading to heterogeneous results. Furthermore, as NSQIP abstracts data from multiple hospitals and providers, there was no standardized post-operative management plan (such as ERAS pathways), which is likely to have further contributed to the heterogeneity of the results. We did not make a distinction between patients who underwent an open versus a robotic approach. However, previous studies have demonstrated that the 30 day re-operation rate following a robotic cystectomy was $5.0 \%,{ }^{18}$ which is similar to out reported rate of $5.8 \%$. 


\section{Conclusions}

Following RC, 5.8\% of bladder cancer patients undergo reoperation within 30 days. Increasing BMI, history of COPD and African American race are factors independently associated with increased risk of reoperation. Reoperation within 30 days of RC is also associated with significantly increased mortality rates, in addition to various other adverse health consequences. These results highlight the need for identifying at-risk patient demographics and pre- and post-operative optimization of those with modifiable risk factors in order to minimize post-operative complications.

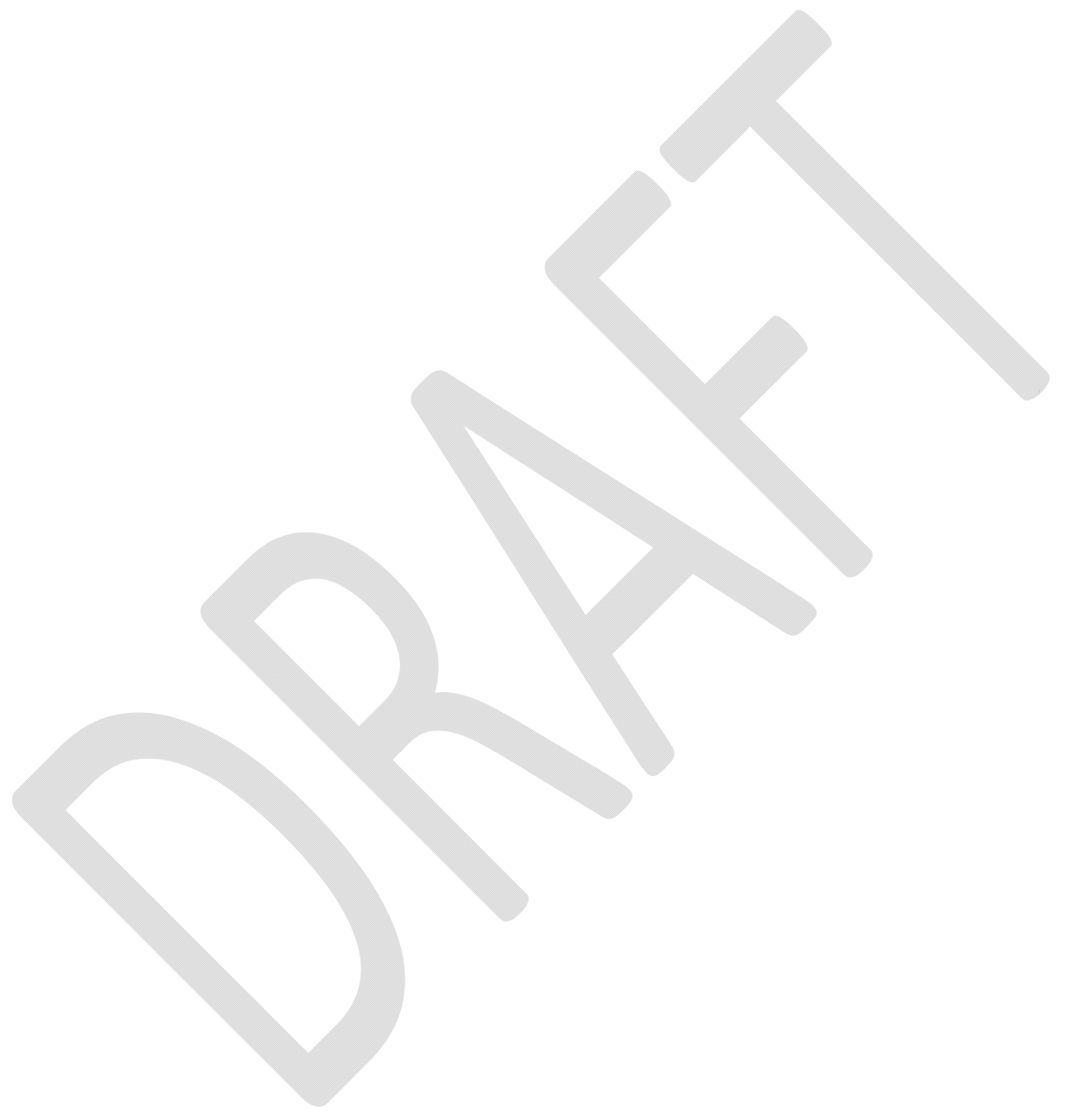




\section{References}

1. Witjes JA, Bruins M, Comperat NC, et al. Muscle-invasive and Metastatic Bladder Cancer. https://uroweb.org/guideline/bladder-cancer-muscle-invasiveand-metastatic/. Accessed February 16, 2019.

2. Babjuk M, Burger M Comperat E, et al. Non-muscle-invasive Bladder Cancer. https://uroweb.org/guideline/non-muscle-invasive-bladder-cancer/._Accessed February 16, 2019.

3. Gandaglia G, Varda B, Sood A, et al. Short-term perioperative outcomes of patients treated with radical cystectomy for bladder cancer included in the National Surgical Quality Improvement Program (NSQIP) database. Can Urol Assoc J 2014;8(9-10):E681-7. https://doi.org/10.5489/cuaj.2069

4. Lyon TD, Boorjian SA, Shah PH, et al. Comprehensive characterization of perioperative reoperation following radical cystectomy. Urol Oncol 2019;S1078-1439(18)30491-5. https://doi.org/10.1016/j.urolonc.2018.11.023

5. American College of Surgeons. ACS NSQIP. https://www.facs.org/qualityprograms/acs-nsqip/about. Accessed February 16, 2019.

6. Arora K, Hanson K, Habermann E, et al. Early Complications and Mortality following Radical Cystectomy: Associations with Malnutrition and Obesity. Bladder Cancer 2018;4(4):377-388. https://doi.org/10.3233/BLC-180173

7. Al-Daghmin A, Aboumohamed A, Din R, et al. Readmission after robotassisted radical cystectomy: outcomes and predictors at 90-day follow-up. Urology 2014;83(2):350-6. https://doi.org/10.1016/j.urology.2013.09.056

8. Lee JL, Oh ES, Lee RW, Finucane TE. Serum Albumin and Prealbumin in Calorically Restricted, Nondiseased Individuals: A Systematic Review. Am J Med 2015;128(9):1023. https://doi.org/10.1016/j.amjmed.2015.03.032

9. Itoh M, Tsuji $\mathrm{T}$, Nemoto $\mathrm{K}$, et al. Undernutrition in patients with COPD and its treatment. Nutrients 2013;5(4):1316-35. https://doi.org/10.3390/nu5041316

10. Lumb AB. Pre-operative respiratory optimization: an expert review. Anesthesia 2019;74 Suppl 1:43-48. https://doi.org/10.1080/14779072.2019.1625771

11. Sathianathen NJ, Weight CJ, Jarosek SL, Konerty BR. Increased Surgical Complications in Smokers Undergoing Radical Cystectomy. Bladder Cancer 2018;4(4):403-409. https://doi.org/10.3233/blc-180185

12. DeDeugd C, Miyake M, Palacios DA, Rosser CJ. The Influence of Race on Overall Survival in Patients with Newly Diagnosed Bladder Cancer. J Racial Ethn Health Disparities 2015;(1):124-31. https://doi.org/10.1007/s40615-0140055-X

13. Klaassen Z, DiBianco JM, Jen RP, et al. Female, Black, and Unmarried Patients Are More Likely to Present With Metastatic Bladder Urothelial Carcinoma. Clin Genitourin Cancer 2016;14(5):e489-e492. https://doi.org/10.1016/j.clgc.2016.04.006

14. Audenet F, Sfakianos JP, Waingankar N, et al. A delay $\geq 8$ weeks to neoadjuvant chemotherapy before radical cystectomy increases the risk of upstaging. Urol Oncol 2019;37(2):116-122. https://doi.org/10.1016/j.urolonc.2018.11.011

15. Johnson DC, Nielsen ME, Matthews J, et al. Neoadjuvant chemotherapy for bladder cancer does not increase risk of perioperative morbidity. BJU Int 2014;114(2):221-8. https://doi.org/10.1111/bju.12585 
16. Enestvedt CK, Diggs BS, Cassera MA, et al. Complications nearly double the cost of care after pancreaticoduodenectomy. Am J Surg 2012;204(3):332-8. https://doi.org/10.1016/j.amjsurg.2011.10.019

17. Ljunqvist O. ERAS - enhanced recovery after surgery: moving evidencebased perioperative care to practice. JPEN J Parenter Enteral Nutr 2014;38(5):559-66. https://doi.org/10.1177/0148607114523451

18. Hussein AA, Hashmi Z, Dibaj S, et al. Reoperations following Robot-Assisted Radical Cystectomy: A Decade of Experience. J Urol 2016. 195(5):13681376. https://doi.org/10.1016/j.juro.2015.10.171

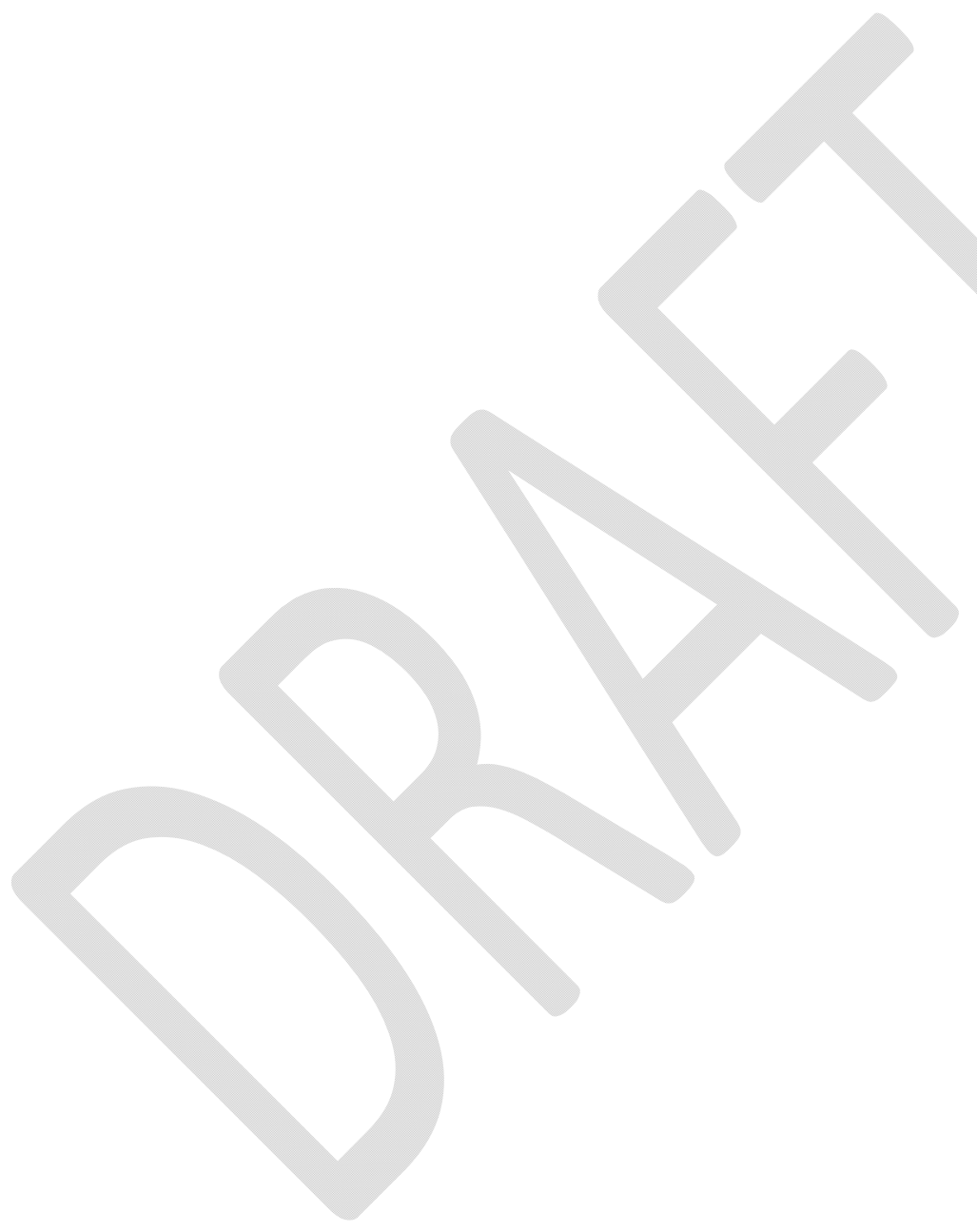




\section{Figures and Tables}

\begin{tabular}{|c|c|c|c|}
\hline Variable & $\begin{array}{c}\text { Patients with } \\
\text { reoperation } \\
(n=152)\end{array}$ & $\begin{array}{c}\text { Patient without } \\
\text { reoperation } \\
(n=2456) \\
\end{array}$ & $\mathbf{p}^{\mathbf{a}}$ \\
\hline Age in years, median (IQR) & $69.0(61.0-76.0)$ & $69.0(61.0-76.0)$ & 0.88 \\
\hline Gender, $\mathrm{n}(\%)$ & & & 0.40 \\
\hline Male & $129(84.9 \%)$ & $2,019(82.2 \%)$ & \\
\hline Female & $23(15.1 \%)$ & $437(17.8)$ & \\
\hline Race, n (\%) & & $x^{2}$ & $0.04^{*}$ \\
\hline African-American & $12(7.9 \%)$ & $98(4.0 \%)$ & \\
\hline Caucasian & $133(87.5 \%)$ & $2,189(89.1 \%)$ & \\
\hline Other & $7(4.6 \%)$ & $169(6.9 \%)$ & \\
\hline BMI in $\mathrm{kg} / \mathrm{m}^{2}$, median (IQR) & $29.1(25.2-33.2)$ & $27.8(24.7-31.4)$ & $0.02^{*}$ \\
\hline ASA, n $(\%)$ & & 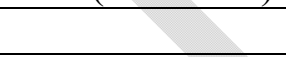 & 0.17 \\
\hline 1 & $0(0 \%)$ & $5(0.2 \%)$ & \\
\hline 2 & $30(19.7 \%)$ & $537(21.9 \%)$ & \\
\hline 3 & $107(70.4 \%)$ & $1,775(72.3 \%)$ & 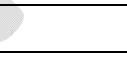 \\
\hline 4 & $15(9.9 \%)$ & $139(5.7 \%)$ & \\
\hline Cardiac history, $\mathrm{n}(\%)$ & $8(5.3 \%)$ & $107(4.4 \%)$ & 0.60 \\
\hline Neurologic history, n (\%) & $0(0 \%)$ & $26(1.1 \%)$ & 0.40 \\
\hline History of COPD, n (\%) & $25(16.5 \%)$ & $179(7.3 \%)$ & $<0.01^{*}$ \\
\hline Diabetes mellitus, n (\%) & $31(20.4 \%)$ & $495(20.2 \%)$ & 0.94 \\
\hline Dialysis, $\mathrm{n}(\%)$ & $0(0 \%)$ & $6(0.2 \%)$ & 0.70 \\
\hline Active smoking, $\mathrm{n}(\%)$ & $47(30.9 \%)$ & $605(24.6 \%)$ & 0.08 \\
\hline Chronic steroid use, n (\%) & $9(5.9 \%)$ & $80(3.3 \%)$ & 0.08 \\
\hline $\begin{array}{l}\text { Preoperative chemotherapy, } n \\
(\%)\end{array}$ & $2(1.3 \%)$ & $77(3.1 \%)$ & 0.20 \\
\hline $\begin{array}{l}\text { Preoperative radiotherapy, } \mathrm{n} \\
(\%)\end{array}$ & $0(0 \%)$ & $2(0.1 \%)$ & 0.89 \\
\hline $\begin{array}{l}\text { Preoperative serum albumin } \\
\text { in } \mathrm{g} / \mathrm{dL} \text {, median (IQR) }\end{array}$ & $4.0(3.7-4.3)$ & $4.0(3.7-4.3)$ & 0.24 \\
\hline $\begin{array}{l}\text { Preoperative functional } \\
\text { status, } n(\%)\end{array}$ & & & 0.67 \\
\hline Independent & $150(98.7 \%)$ & $2412(98.2 \%)$ & \\
\hline Partially/totally dependent & $2(1.3 \%)$ & $44(1.8 \%)$ & \\
\hline Urinary diversion, $\mathrm{n}(\%)$ & & & 0.09 \\
\hline Ileal conduit & $129(84.5 \%)$ & $1,944(79.2 \%)$ & \\
\hline Continent diversion & $23(15.1 \%)$ & $512(20.9 \%)$ & \\
\hline
\end{tabular}

aUsing Chi-squared or Fisher's exact test for categorical variables and student's t-test for continuous variables. ${ }^{*}$ Denotes $p<0.05$. ASA: American Society of Anesthesiologists; BMI: body mass index; COPD: Chronic obstructive pulmonary disease; IQR: interquartile range. 


\begin{tabular}{|c|c|c|}
\hline Variable & Odds ratio & $95 \%$ confidence interval \\
\hline Age & 1.01 & $0.99-1.03$ \\
\hline Gender (male vs. female) & 1.34 & $0.84-2.13$ \\
\hline Race (African American vs. Caucasian) & 2.29 & $1.21-4.34$ \\
\hline Race (Other vs. Caucasian) & 0.70 & $0.31-1.52$ \\
\hline Body mass index & 1.04 & $1.02-1.07$ \\
\hline ASA (2 vs. 1) & 0.95 & $0.62-1.46$ \\
\hline History of diabetes mellitus & 0.88 & $0.57-1.34$ \\
\hline Active smoking & 1.46 & $0.99-2.17$ \\
\hline History of COPD & 2.33 & $1.45-3.74$ \\
\hline Chronic steroid use & 1.75 & $0.85-3.63$ \\
\hline $\begin{array}{l}\text { Functional status (partially/totally } \\
\text { dependent vs. independent) }\end{array}$ & 0.72 & $0.17-3.11$ \\
\hline $\begin{array}{l}\text { Type of diversion (continent vs. ileal } \\
\text { conduit) }\end{array}$ & 0.73 & $0.45-1.18$ \\
\hline
\end{tabular}

ASA: American Society of Anesthesiologists; COPD: chronic obstructive pulmonary disease. 


\begin{tabular}{|l|c|c|c|}
\hline \multicolumn{4}{|c|}{ Table 3. Adverse events following radical cystectomy for bladder cancer } \\
patients with and without reoperation within 30 days of surgery \\
\hline Adverse events & $\begin{array}{c}\text { Patients with } \\
\text { reoperation } \\
\text { (n=152) }\end{array}$ & $\begin{array}{c}\text { Patient without } \\
\text { reoperation } \\
\text { (n=2456) }\end{array}$ & p $^{\mathbf{a}}$ \\
\hline Mortality & $6(4.0 \%)$ & $38(1.6 \%)$ & $0.03^{*}$ \\
\hline Cardiac event & $11(7.2 \%)$ & $46(1.9 \%)$ & $<0.01^{*}$ \\
\hline Neurologic event & $3(2.0 \%)$ & $12(0.5 \%)$ & $0.02^{*}$ \\
\hline Pulmonary event & $35(23.0 \%)$ & $73(3.0 \%)$ & $<0.01^{*}$ \\
\hline $\begin{array}{l}\text { Venous thromboembolic } \\
\text { event }\end{array}$ & $16(10.5 \%)$ & $132(5.4 \%)$ & $0.01^{*}$ \\
\hline DVT & $9(5.9 \%)$ & $89(3.6 \%)$ & 0.15 \\
\hline PE & $9(5.9 \%)$ & $59(2.4 \%)$ & $0.01^{*}$ \\
\hline Any infection & $98(64.5 \%)$ & $593(24.1 \%)$ & $<0.01^{*}$ \\
\hline Sepsis & $66(43.4 \%)$ & $293(11.9 \%)$ & $<0.01^{*}$ \\
\hline Pneumonia & $20(13.2 \%)$ & $69(2.8 \%)$ & $<0.01^{*}$ \\
\hline Urinary tract infection & $22(14.5 \%)$ & $210(8.6 \%)$ & $0.013^{*}$ \\
\hline Wound infection & $73(48.0 \%)$ & $271(11.0 \%)$ & $<0.01^{*}$ \\
\hline Superficial SSI, n (\%) & $20(13.2 \%)$ & $(143(5.8 \%)$ & $<0.01^{*}$ \\
\hline Deep incisional SSI, n (\%) & $17(11.2 \%)$ & $34(1.4 \%)$ & $<0.01^{*}$ \\
\hline Organ space SSI, n (\%) & $40(26.3 \%)$ & $108(4.4 \%)$ & $<0.01^{*}$ \\
\hline $\begin{array}{l}\text { Prolonged hospital length of } \\
\text { stay, n (\%) }\end{array}$ & $124(81.6 \%)$ & $1164(47.4 \%)$ & $<0.01^{*}$ \\
\hline Length of stay, median (IQR) & $16.5(8.0-26.5)$ & $7.0(6.0-10.0)$ & $<0.01^{*}$ \\
\hline Using Chisgar $)$ & & & \\
\hline
\end{tabular}

${ }^{a}$ Using Chi-squared or Fisher's exact test for categorical variables and student's t-test for continuous variables. "Denotes $p<0.05$. DVT: deep vein thrombosis; IQR: interquartile range; PE: pulmonary embolism; SSI: surgical site infection. 